\title{
A two part, multi-centre, multiple dose study of Erythrocyte Encapsulated Thymidine Phosphorylase (EETP) in patients with Mitochondrial Neurogastrointestinal Encephalomyopathy (MNGIE)
}

Niranjanan Nirmalananthan ${ }^{1}$ (niranjanan.nirmalananthan@stgeorges.nhs.uk), Michelle Levene ${ }^{2}$, Massimiliano Filosto ${ }^{3 *}$, Thomas Klopstock ${ }^{4 *}$, Cornelia Kornblum ${ }^{5 *}$, Hanna Mandel ${ }^{6 *}$, Shamima Rahman ${ }^{7 *}$, Agathe Roubertie ${ }^{8 *}$, Mauro Scarpelli3*, Orphan Technologies Ltd ${ }^{9}$ and Bridget E. Bax ${ }^{2}$

${ }^{*}$ Authors contributed equally

${ }^{1}$ St Georges University Hospitals NHS Foundation Trust, London; UK; 2 St George's University of London, UK ${ }^{3}$; University Hospital "Spedali Civili", Brescia, Italy; ${ }^{4}$ University of Munich, Germany; ${ }^{5}$ University of Bonn, Germany; ${ }^{6}$ Galilee Medical Center, Israel; ${ }^{7}$ University College London, UK; ${ }^{8}$ Centre Hospitalier Universitaire de Montpellier, France;

${ }^{9}$ Rapperswil, Switzerland

Background: MNGIE is a fatal inherited disease caused by mutations in the gene encoding thymidine phosphorylase, leading to reduced enzyme activity, systemic toxic accumulation of nucleosides and secondary mitochondrial DNA damage. Enzyme replacement with EETP has demonstrated metabolic and clinical improvements in MNGIE in our compassionate treatment programme.

Aims: To determine the long-term safety, tolerability, pharmacodynamics and clinical efficacy of repeated doses of EETP in patients with MNGIE.

Methods: This multi-centre, multiple dose study will be conducted in two parts: a double-blind, multiple-ascending dose phase (Part 1) of eight weeks and an open-label, multiple-dose phase (Part 2) of 24 months. We will recruit 12 patients who meet the following criteria: $\geq 18$ years of age, a confirmed diagnosis of MNGIE and able to adequately grant informed consent. In Part 1 , patients will be randomised to three dosing cohorts $(20,50$ or $100 \mathrm{IU} / \mathrm{kg})$ at a ratio of $3: 1$ to receive EETP or placebo. Patients randomised to placebo will receive two doses of placebo and then active drug for third and fourth dose on Day 28 and Day 42 . Collective safety and pharmacodynamics data will be reviewed from all 4 patients treated at each dose before dose escalation to the next cohort. Patients completing Part 1 will be entered into Part 2 to receive EETP every 2 weeks, where the dose may be escalated where pharmacodynamics data indicates the patient has not achieved metabolic correction.

Results: Mean laboratory values over time will be plotted by dose group. Descriptive statistics will be provided for clinical laboratory data, pharmacodynamics data and vital signs. Associations between pharmacodynamics parameters and clinical activity parameters will be explored. Data collection should be finalised by March 2020.

Conclusions: Positive results from this trial will support a Marketing Authorisation Application for EETP and its therapeutic use in treating patients with MNGIE. 